\title{
THE
}
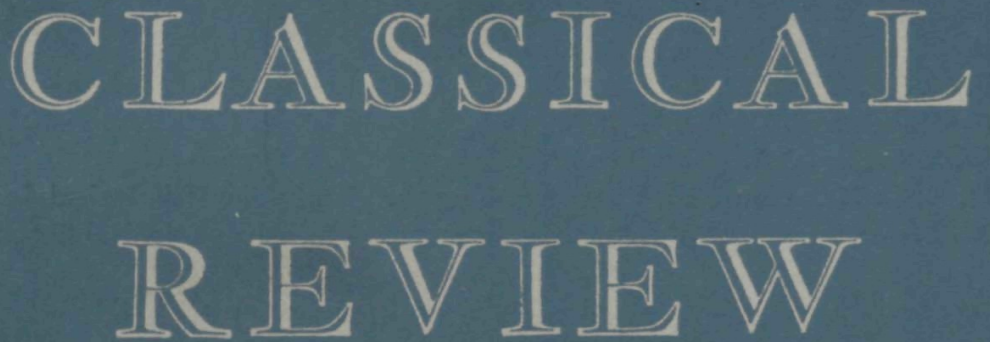

NEW SERIES VOLUME I NUMBERS 3,4 (Vol. Lxv of the continuous series)

\section{December $195^{I}$}

OXFORD

\section{AT THE CLARENDON PRESS}

LONDON NEW YORK TORONTO MELBOURNE

GEOFFREY CUMBERLEGE : OXFORD UNIVERSITY PRESS

IOS. Einiasura net. Yearly subscription, 16 s, net, post free. Combined yearly subscription for the

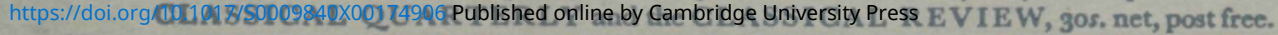




\section{THE CLASSICAL REVIEW}

Editors C. J. ForDYGE, M.A., 3 The University, Glasgow, W. 2

All communications intended for the Editors should be sent to Mr. Fordyce. Books for review should be sent to the Publisher, Geoffrey Cumberlege, Oxford Universily Press, Amen House, Warwick Square, London, E.C. 4 .

BOARD OF MANAGEMENT

Professor E. H. Warmington, M.A. (Chairman)

Professor R. G. Austin, M.A. (Hon. Treas.)

F. H. Sandbach, M.A.

Professor F. W. Walbank, M.A.

W. S. Watt, M.A. (Hon. Sec.)

Representing the

Council of the

Classical Association

N. G. L. Hammond, M.A., representing the Cambridge Philological Society

M. Platnauer, B.Litt., M.A., representing the Oxford Philological Society

With the co-operation of

Professor B. D. Meritt, Institute for Advanced Study, Princeton,

Professor J. A. FitzHerbert, University of Adelaide, and

Professor Homer A. Thompson, Institute for Advanced Study, Princeton

\section{New Series Volume I Nos. 3, $4 \quad$ Dec. $1955^{I}$ CONTENTS}

"A

A. Y. CAMPBELL 129

Dactyls in Comic Trochaics

M. PLATNAUER I 32

Aegisthus and the Chorus

Notes on Thucydides

A. D. FITTON BROWN 133

Varia Iuvenaliana

A. W. GOMME I 35

The Function of Tense Variation in the Subjunctive Mood of Oratio Obliqua

J. G. GRIFFITH I 38

Herodotus vi. 74

Libanius, Or. xxviii. 20

Two Notes on Naevius

M. ANDREWES 142

D. HEREWARD I 46

A. F. NORMAN 146

o. SKUTSCH 146

\section{REVIEWS :}

Aeschylus, Agamemnon (Fraenkel), R. P. wrnnnscton-1NGram, 147; The Sophocles Reunsion of Moschopulos (Turyn), Démétrius Triclinius et les recensions médiévales de Sophocle (Aubreton), R. M. RATtenBury, 151 ; Euripides, Helena (Campbell), D. W. LuCAs, 154 ; The Greek Philosophers from Thales to Aristotle (Guthrie), J. TATE, 156; Greek Philosophy (de Vogel), G. B. KERFERD, 157; Platon (Hoffmann), G. B. KERFERD, 159; The Unieritten Philosophy (Cornford), D. J. ALLAN, 160; Platonis Phaedo interprete Aristippo (Minio-Paluello), D. A. REes, r6r; Stoa und Stoiker (Pohlenz), G. B. KerFerd, 163; Theocritus (Gow), M. Platnauer, 164; Onesicritus (Brown), G. T. GRIFFTH, 169; Storia della letteratura latina (Paratore), R. BRownswg, 17x; Nacuius poeta (Marmorale), o. sKuTscH, 174; Lucretius translated (Brown), G. c. wHrттіск, 177; Die Dichtkanst Virgils (Pöschl), M. L. CLARKE, I 78; Horati opera (Klingner), T. E. WRiGHT, 180 ; Phedre et ses Fables (Herrmann), c. J. FORDYce, 182; Cesar, Guerre d'Afrique (Bouvet), c. o. BRINK, 183; Aṕpendix Sallustiana (Kurfess), G. c. whrrtick, 185; Ciceronis Epistulae ad Familiares (Moricca), w. s. wATT, 186; Ciceronis De Offriis (Atzert), A. G. LEE, 189; Cicerone, de Re Publica (Ferrero), с. H. POYser, 190; Pline, Histoire Naturelle, xii (Ernout), R. G. AUstrs, r91; Petronius, Cena Trimalchionis (Sedgwick), R. Brownivg, 193; The Greater Roman Historians (Laistner), R. SYME, 194; Roman Declamation (Bonner), c. J. FordycE, 197; Minucii Felicis Octavius (Pellegrino, Quispel), R. Browning, 198 ; St. Augustine's De Musica (Knight), R. P. wiNNINOTON-INGRAM, 200; Untersuchungen über das lateinische Gerundium und Gerundivum 


\title{
THE
}

\section{L A S S I C A L RE V IE W}

\author{
EDITED BY \\ C. J. FORDYGE \\ AND \\ R. M. RATTENBURY
}

BOARD OF MANAGEMENT

Prop. E. H. WARMINGTON, M.A. (Chairman)

Prof. R. G. AUSTIN (Hon. Treasurer)

F. H. SANDBACH, M.A.

Prof. F. W. WALBANK, M.A.

W. S. WATT, M.A. (Hon. Secretary)

N. G. L. HAMMOND, M.A. (Representing the Cambridge Philological Society)

M. PLATNAUER, B.Litr., M.A. (Representing the Oxford Philological Society)

With the co-operation of

Prof. B. D. MERITT, Institute of Advanced Study, Princeton

Pror. J. A. FITZHERHERT, University of Adelaide, and

Prof. HOMER A. THOMPSON, Institute of Advanced Study, Princeton

NEW SERIES

VOLUME I

(VOL. LXV OF THE CONTINUOUS SERIES)

Published in co-operation with the Classical Association OXFORD: AT THE GLARENDON PRESS

I95 I 
Oxford University Press, Amen House, E.C. 4 GLASGOW NEW YORK TORONTO MELBOURNE WELLINGTON BOMBAY CALCUTTA MADRAS CAPE TOWN

Geoffrey Cumberlege, Publisher to the University 


\section{TABLE OF CONTENTS}

\section{Number I}

Latin amata, amita

A. C. MOORHOUSE

Juvenal I o. I $73^{-8}$

J. O. THOMSON 3

The Will of $Q$. Veranius

C. E. STEVENS 4

Claudius and the Orcades

The Homeric Epigram to the Potters

Cicero, Pro Milone 98

A Pun in Suetonius

In Summa

Some Manuscripts of Juvenal

C. E. STEVENS 7

R. M. COOK 9

W. L. GRANT 9

T. L. ZINN IO

D. B. GREGOR IO

F. H. SANDBACH II

REVIEWS:

Anthologia Lyrica Graeca, I (Diehl), D. L. PAGE, I I; De duobus Alcaei carminibus, De duobus Sapphus carminibus (Steffen), D. L. PAGE, 14; Bacchylidis carmina (Snell), D. S. ROBERTSON, I5; Pindare et Platon (Des Places), J. TATE, I 7 ; De Rheso tragoedia (Sneller), D. W. Lucas, I8; Demosthenes, vol. VII (DeWitt), J. TATE, 20; Xénophon, Économique (Chantraine), G. B. KERFERD, 21; The Philosophy of Plato (Field), D. J. Allan, 23; An Index to Aristotle (Organ), D. A. REES, 24; Aristotelis Categoriae et Liber de Interpretatione (Minio-Paluello), D. A. REES, 26; Zwei Aristotelische Frïhschriften über die Ideenlehre (Wilpert), D. J. ALLAN, 27; Storia della Letteratura Latina, I (Rostagni), R. BRownING, 29; Iambic Words and Regard for Accent in Plautus (Harsh), W. A. LAIDLAW, 32 ; I'Poetae Novelli' (Castorina), R. BRownING, 34; Letters of Cicero (Wilkinson), w. s. WATt, 36; Constancy in Livy's Latinity (Gries), G. G. WhrtTiGK, 37; Per la Storia dei Municipi (Manni), R. MEIGGs, 38; Documents Illustrating the Reigns of Augustus and Tiberius (Ehrenberg and Jones), J. P. v. D. BALsDon, 40; The Classical Tradition (Highet), J. A. K. THOMSON, 42; Some School-Books, D. s. Colman, 45; Mycenae (Wace), R. W. hutchinson, $4^{8}$.

Short Reviews

Summaries of Periodicals

Books Received

\section{Number 2}

Pindar, Isthmians 6. 4 K. J. DOVER 65 Plato, Phaedo 67 c 5 J. V. LUGE 66

The Rhetra of Lycurgus

J. L. MYRES 67

Two Passages of Lucan

A Transposition in Statius A. HUDSON-WILLIAMS 68

Two Notes on Juvenal

G. P. GOOLD 7I

An Emendation in Isocrates

W. GLAUSEN 73

T. L. ZINN 74

The 'Murder' of Drusus, Son of Tiberius

J. P. V. D. BALSDON 75

A propos d'un compte rendu (The Budé Theognis) J. CARRIÈRE 75 REVIEWS:

Callimachus, I (Pfeiffer), E. A. BARBer, 78; Oxyrhynchus Papyri, XIX, E. A. BARBER, 80; Atthis (Jacoby), A. W. GOMME, 82; Plato's Life and Thought (Bluck), J. B. sKEMP, 86; Kleanthes van Assos (Verbeke), J. TATE, 88; The Latters of Alciphron, Aelian, and Philostratus (Benner and Fobes), H. Ll. hudson-williams, 9o; Cicero, Pro Roscio Amerino, In Verrem, iv-v (Klotz), Pro Sulla (Karsten), Pro Archia (Reis), J. H. sImon, 92; Symbola Coloniensia Iosepho Kroll oblata, J. A. Davinson, 93; Geschichte der Autobiographie, I (Misch), w. s. Magunvness, 95; Supplementum Epigraphicum Graecum, X, R. MEIGgs, 96; Ancient Sparta (Chrimes), F. w. wALBANk, 98; Alexander der Grosse (Schachermeyr), A. R. BURN, 100; Constantin der Grosse (Vogt), A. R. BURN, I02; Das Urchristentum (Bultmann), T. w. MANson, I03; Vom Ursprung und Ziel der Geschichte (Jaspers), J. L. MYREs, IO4; History of Greek Religion, The Minoan-Mycenaean Religion and its Survival (Nilsson), w. K. C. GUTHRIE, I05; Vesta (Brelich), H. J. ROSE, 107; Archaic Greek Art (Richter), M. ROBERTSON, IO9; Das hellenistische Bildnis (Buschnor), A. W. LAWRENCE, III; Greek Altars (Yavis), A. W. LAWRENCE, II 2. 
Summaries of Periodicals $\quad$ I22

$\begin{array}{ll}\text { Notes and News } & 124\end{array}$

Books Received I 26

Numbers 3, 4

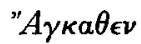

A. Y. CAMPBELL 129

Dactyls in Comic Trochaics

M. PLATNAUER I 32

Aegisthus and the Chorus

A. D. FITTON BROWN 133

Notes on Thucydides

A. W. GOMME I 35

Varia Iuvenaliana

J. G. GRIFFITH I 38

The Function of Tense Variation in the Subjunctive Mood of Oratio

Obliqua

M. ANDREWES I 42

Herodotus vi. 74

D. HEREWARD I 46

Libanius, Or. xxviii. 20

A. F. NORMAN 146

Two Notes on Naevius

o. SKUTSGH

146

\section{REVIEWS:}

Aeschylus, Agamemnon (Fraenkel), R. P. WinningTon-ingram, 147; The Sophocles Recension of Moschopulos (Turyn), Demétrius Triclinius et les recensions médietuales de Sophocle (Aubreton), R. M. RATtenbURY, I5I ; Euripides, Helena (Campbell), D. W. LUCAS, I 54; The Greek Philosophers from Thales to Aristotle (Guthrie), J. TATE, I 56 ; Greek Philosophy (de Vogel), a. B. KERFERD, I57; Plato (Hoffmann), G. B. KERFerD, I59; The Unwritten Philosophy (Cornford), D. J. ALLAN, I6o; Platonis Phaedo interprete Aristippo (Minio-Paluello), D. A. REES, 16r ; Stoa und Stoiker (Pohlenz), G. B. KERFERD, 163; Theocritus (Gow), M. PLATNAUER, 164; Onesicritus (Brown), G.T. GRIFFTH, I69; Storia della Letteratura Latina (Paratore), R. BRowNING, I 7I ; Naevius poeta (Marmorale), o. SKUTSGH, I 74; Lucretius translated (Brown), G. c. WHrrTICK, 177; Die Dichtkunst Virgils (Pöschl), M. L. CLARke 178; Horati opera (Klingner), T. E. WRIGHT, 180; Phèdre et ses Fables (Herrmann), c. J. FoRDYGe, 180; César, Guerre d'Afrique (Bouvet), c. o. BRINK, 183; Appendix Sallustiana (Kurfess), G. c. WhrrTrck, I85; Ciceronis Epistulae ad Familiares (Moricca), w. s. watT, I86; Ciceronis De Officiis (Atzert), A. G. LEE, I89; Cicerone, De Re Publica (Ferrero), G. H. Poyser; 190; Pline, Histoire Naturelle, xii (Ernout), R. G. Austrin, I9I; Petronius, Cena Trimalchionis (Sedgwick), R. BROWNING, I93; The Greater Roman Historians (Laistner), R. SYME, 194; Roman Declamation (Bonner), c. J. FORDYCE, 197; Minucii Felicis Octavius (Pellegrino, Quispel), R. BRoWnIng, 198; St. Augustine's De Musica (Knight), R. P. WInningtonINGRAM, 200; Untersuchungen über das lateinische Gerundium und Gerundivum (Aalto), D. M. JONES, 201; Les termes de couleur dans la langue latine (André), E. LAUGHTON, 201; Handbuch der Archöologie, iv (Otto-Herbig), F. H. stubBings, 205; Metallurgy in Antiquity (Forbes), A. w. LAWRENCE, 207; The Greeks and their Gods (Guthrie), H. J. ROSE, 208; Mythos (Gitti), H. J. ROSE, 210 ; Die Kabiran (Hemberg), H. J. ROSE, 211 ; Studies in Magical Amulets (Bonner), H. J. ROSE, 213 ; Les premieres civilisations (Chapouthier, Aymard), T. J. DUNBABIN, 214; Greek City-States (Freeman),

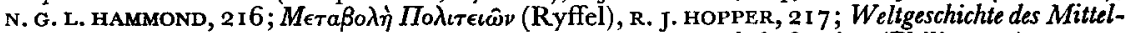
meer-Raumes (Kornemann), M. CARY, 219; Die griechischen Landschaften, i. I (Philippson), N. G. L. HAMMOND, 221 ; Supplementum Epigraphicum Graecum, xi. I (Hondius), P. M. FRASER, 223; Ainos, its History and Coinage (May), c. T. SELTMAN, 225; Coins of the Roman Empire in the British Museum, v (Mattingly), M. GRANT, 227; Roman Anniversary Issues (Grant), c. M. KRAAY, 229; Aspects of the Principate of Tiberius (Grant), c. H.v. SUTHERLand, 23I; Memoirs of the American Academy at Rome, xix, A. N. SHERWIN-WHITE, 233.

Short Reviews

Summaries of Periodicals

Notes and News

248

Books Received

Index 